PR11.03.12 X-RAY METHOD FOR STRUCTURE DETERMINATION OF EOULLIBRIUM SOLID-LIOUDD SYSTEM. Yu.V. Agrafonov, F.E. Ushakov Department of Physics, Irkutsk State University, Irkutsk, Russia

A universal statistical approach for description of X-ray scattering by substance in any state of matter is suggested. $X$-ray scattering by solid and liquid phases is examined by different ways.

If solid is considered, dimensions of cell, location of lattice sites and electron distribution inside the cell are usually defined. The long-range order describes crystal state: unary distribution function defining location of atoms in lattice sites is a periodic one. X-rays are scattered on atomic planes.

For liquid the space distribution of moving particles is defined. The short-range order describing liquid structure is related to the density fluctuation that is completely predetermined by binary distribution function.

In our approach averaging over statistical ensemble leads to expression for scattered radiation intensity that include unary and binary distribution functions. This expression can be applied either for homogeneous or inhomogeneous systems.

When crystal is in equilibrium with its molten matter (liquid to solid transition), both long-range and short-range orders contribute to the scattering. The information about scattering (intensity of scattered radiation) being obtaining, the distribution functions of high-temperature crystal could be defined. Also the approach can be used for testing phase transition theories.

PR11.03.13 DIPOLE STRUCTURE MODEL OF MICA CRYSTALS. Metsik M.S., Novikov G.K., University of Irkutsk,Russia.

The new structural and energetic basic of the model of mica crystals is the presense of ionic $\mathrm{K}$ and $\mathrm{OH}$ sublattice in matrix of covalent bound up atoms of $\mathrm{Si}, \mathrm{Al}, \mathrm{Mg}, \mathrm{O}$. These atoms form plane element packets.

It is supposed that ions $\mathrm{K}$ and $\mathrm{OH}$ form the system of packet electrical dipoles creating force connections between packets. These forces are on the order of magnitude lower than the ones acting inside of packets.

Consequently kinds of mica are the typical inorganic polimerides with double nature of force connections covalent force is in packets and vandervaals one is between packets.

The model well explains great anisotropy of mechanical properties along two crystallographic directions and perfect crystal cleavage along (001) plane it allows to calculate of energy connection between packets and etc.In can also explain powerful coronoelectret effect with the voltage $=4 \mathrm{Kv}$ relaxing during $\tau \cong 100$ days discovered by us. That effect is conditioned by the capture of charge carriers in equilibrium vacancies of ionic sublattice with energies $0,2 \mathrm{eV}$ and $0,75 \mathrm{eV}$. These values coincide with the calculated ones according to structural data of crystal energy diagramms.

\section{Solid State Reactions:}

\section{Structural Thermodynamics \& Kinetic Aspects}

MS11.04.01 REARRANGEMENT REACTIONS IN SOLID STATE ORGANIC COMPOUNDS. M. Kaftory, E. Handelsman-Benory, M. Botoshansky, Department of Chemistry, Technion Israel Institute of Technology, 32000 Haifa, Israel

Few known examples of rearrangement reactions in the solid state will be briefly described. An interesting example of solid state reaction is the stepwise methyl rearrangement of $2,4,6$ trimethoxy-1,3,5-triazine (I) to 1,3,5-trimethyl-2,4,6trioxohexahydro-s-triazine (IV) through (II) and (III). Studies by MS and NMR $[1,2]$, have shown that the methyl

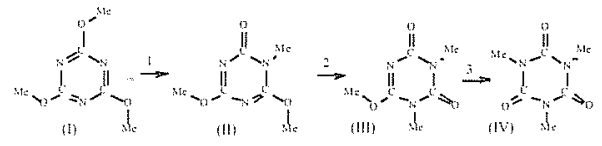

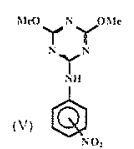

migration in the melt is intermolecular. Also, it was found that some of the rearrangement steps occur in the solid state. We have studied the thermal behavior of each compound by HTXRD, by DSC and analyzed the topochemistry of the rearrangements from their crystal structures. We have found that steps 1 and 3 are taking place in the melt while step 2 in the solid. The crystal structure of (II) shows that methyl migration can take place by two routes: "Homo Chain Mechanism" will lead to compound (III) and "Hetero Ribbon Mechanism" will produce (IV). Thermal behavior studies suggest that both reactions are practically taking place. Kinetic study of the solid state rearrangement found for step 2 will be described.

Compounds of type (V) shown to undergo methyl migration either in the solid state or in the melt. In one of the compounds a color change was observed whose nature is under investigation.

[1] Tosato, M. L. (1982). J. Chem. Soc. Perkin Trans. II, 1321.

[2] Tosato, M. L. (1984), J. Chem. Soc. Perkin Trans. II, 1593.

MS11.04.02 CRYSTAL-TO-CRYSTAL PHOTOREACTIONS: THE WAVE LENGTH DEPENDENCE OF TOPOCHEMICAL REACTIONS. Volker Enkelmann 